\title{
Modification of basal brain metabolism and neuronal activity is an acquired feature of obesity
}

T he prefrontal cortex (PFC) is a region of the brain that is implicated in moderating appropriate

behavior, mediating satiety and also meal termination. In some obese individuals, the PFC is known to be activated to a lesser extent than it is in lean individuals; however, until now, it has not been clear when this abnormality in neuronal activity is established (that is, before an individual becomes obese or as an acquired feature of obesity). In a study published in Obesity, David Val-Laillet and colleagues have demonstrated that modification of basal brain metabolism and the decreased neuronal activity of the PFC is most likely an acquired feature of obesity.

"Because this abnormality disappears in formerly obese individuals who have undergone successful weight loss, we hypothesized that less activation in the PFC is not 'hard wired' in disease-prone subjects, but rather depends on the nutritional context," explains Val-Laillet, corresponding author of the study. As it is difficult to perform controlled studies in obese humans, the researchers used an adult animal model of obesity to investigate whether the abnormal activity in the PFC pre-exists obesity or occurs once a subject is obese.

Pigs and humans have a similar brain structure and pigs can develop known human disorders, such as diabetes and excessive fat deposition. Rather than working with conventional pigs, however, the group chose to use adult minipigs. Adult minipigs are of limited size, which makes them much easier to handle than conventional pigs, and the same medical imaging tools that are used for humans can also be used for minipigs. In addition, the ability to induce obesity in minipigs by using a Western diet enriched with carbohydrates and lipids had already been established and the metabolic, physiological and behavioral consequences of such diet-induced obesity reported.
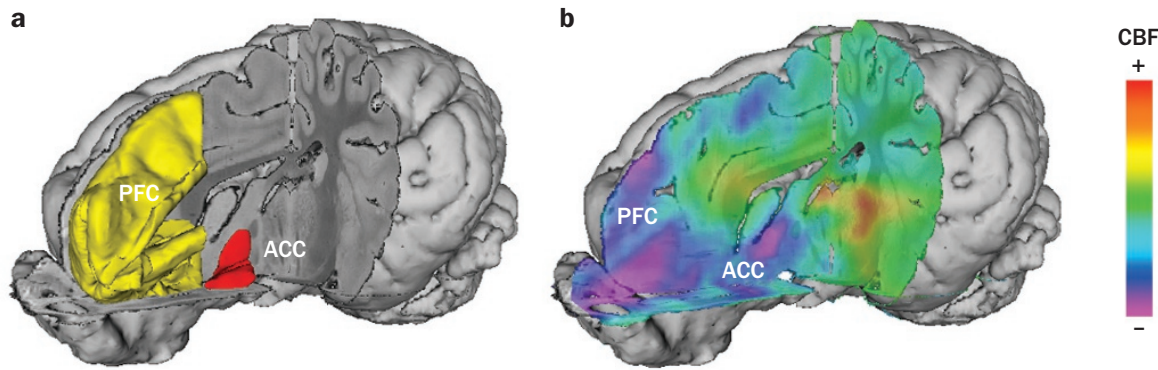

Three-dimensional representations of the pig brain. a | Anatomical localisation of the prefrontal cortex (PFC) and nucleus accumbens (Acc). b | Cerebral blood flow (CBF) - positive CBF values indicate activation and negative values indicate deactivation in obese subjects compared with lean subjects. Courtesy of D. Val-Laillet.

Val-Laillet and colleagues used single photon emission computed tomography (SPECT) to compare basal cerebral blood flow $(\mathrm{CBF})$ - and therefore neuronal activity-in the PFCs of seven dietinduced obese adult and nine lean adult minipigs. Other areas of the brain in which activity differed between the obese and lean minipigs were also identified and these data compared with data obtained in humans. Whether body weight and/or the amount of food consumed the day prior to imaging had any relation to brain activity was also considered.

"This study is the very first to describe the brain metabolism in obese minipigs," says Val-Laillet. "Much more important is the fact that our results were similar in many ways to what has been described in humans. We showed that some brain functional features described in obese humans can also be found in dietinduced obese minipigs, which suggests that the nutritional context is of prime importance in the modulation of the basal brain metabolism and the possible emergence of cerebral functional abnormalities."

One anomaly identified by the researchers was decreased activity in the PFC. They also found an inverse association between body weight and activity in the PFC. In addition, in the obese minipigs activity was reduced in the dopaminergic structures of the ventral tegmental area and nucleus accumbens (Acc). This finding suggested to the authors that the dopaminergic system in the obese minipigs may be altered, as it is in obese humans. As dopaminergic alterations are present in drug-addicted individuals, the researchers speculate about the existence of a food addiction phenomenon.

"Exposure to a palatable and deleterious diet might induce, in minipigs similarly to humans, some kind of habituation and consequently enhance the level of food stimulation required to fulfill the hedonic requirements of the organism," suggests Val-Laillet.

The researchers believe the minipig model will be useful for further studies of decreased PFC activity and potential therapies. "In the future, the minipig model might be used to experiment with new therapeutic strategies aimed at regulating eating behavior and body weight $\ldots$ before being applied to obese humans," concludes Val-Laillet.

Natalie J. Wood

Original article Val-Laillet, D. et al. Changes in brain activity after a diet-induced obesity. Obesity doi:10.1038/ oby.2010.292

Further reading Val-Laillet, D. et al. Chronic vagus nerve stimulation decreased weight gain, food consumption and sweet craving in adult obese minipigs. Appetite 55, 245-252 (2010) 